\title{
Effective Lifestyles for a Healthy Life above Forty Years
}

\author{
Matthew Chukwuma Michael* \\ Department of Mathematics and Statistics, Nigeria
}

*Corresponding author: CMatthew Chukwuma Michael, Department of Mathematics and Statistics, School of Applied Sciences, Delta State Polytechnic, Ogwashi-Uku, Nigeria.

Received Date: December 24, 2018

Published Date: January 11, 2019

\begin{abstract}
This paper seek to identify the effective lifestyles that enhance a healthy life above forty years of age. It highlighted and explained some age-related health issue and lucidly showed how they vary with age. It viewed lifestyle from the perspective of a cultural way of doing things and the individualistic perspective as those individual habits that may be relatively distinct from the general way. It discussed some effects of lifestyle and age on health. It concluded that effective lifestyles for healthy life above forty years are those lifestyles that should be adopted in order to achieve a complete wellbeing of the individual in that age group. It finally highlighted them to include, but not limited to, proper choice of what to eat and what to avoid; proper choice of life partners; regular, enjoyable and satisfactory sexual life; planning of economic and financial life; avoiding illicit drugs and drug/substance abuse and adopting good stress management strategies.
\end{abstract}

\section{Introduction}

Life is a process of observing, experiencing and performing the characteristics of life which include; feeding/nutrition, growth, excretion, irritability, movement, respiration, reproduction and ultimately, death. It is easier to explain the act of living than to explain life itself. We experience the characteristics of life on daily basis except one (Death) which we experience once in a life time. Funny as it may, but obvious, people are scared of death and often times, do not want/pray to experience it. However, death is part of the events that define life; hence, all living organisms die in order to complete their life journey. Health is a state of general mental, physical, psychological, social and economic wellbeing of an individual. Therefore, health of an individual is measured by the quality of life he/she lives and how useful the individual is to the society.

Age, as they say, is just a number but a saying goes that a fool at forty is a fool forever. More so, for every functional process, there is a period for optimum performance, wear and tear, repair and, finally, breakdown. Living organisms are not exceptions; from birth, many events occur in the transition to death and, those events, most times, depend on age. Most times, the longevity and functionality of a system/process depends to a large extent on the way it is operated/handled or managed. What increases longevity is ensuring that what promotes good standard and specifications of a system are upheld. To the human organism, the way we observe, experience and perform the characteristics of life are called lifestyles. Where the lifestyles are found to meet up with good standards and specifications, they are said to be effective. Thus, it is not out of place to examine the effective lifestyles that will enhance healthy life at forty years and beyond.

\section{Age Related Health Issue}

Most events in the journey of life, as stated earlier are age dependent. At birth, babies are completely under the care of their parents and adults around them. Their immune system is very weak and they only have to suck the colostrum of their mother's breast in order to build their body immunity. Their immune system is built rapidly to an optimal level until a certain age from where it gradually deteriorates to a zero level.

How often we fall sick, the amount of economic and social pressure on us, the emotional tension we have to face, the number of mental stress we pass through, the wear and tear at moveable joints, the flow rate of body fluids, growth of tissues, to mention just a few, are dependent on age. 
How often we fall sick and the type of sickness we experience are functions of age. The way we eat, for instance, changes with age. What we eat is also a function of age. It is common place that while youths drink alcohol heavily, adults consume regularly. While youths are involved in more physical exercises, adults are involved in more mental exercises. While youths experience heartbreak in the form of separation with their lovers, adults find embrace heartbreak in the form of divorce, separation or death of a partner. Hence, heartbreak is temporary in youths but more perpetual in adults. Diseases are known to affect all individuals irrespective of age but infectious diseases are prevalent among youths while more permanent and irreversible diseases occur at older age. Examples are cancers, prostatitis, and organ damage.

The types of people an individual mixes up with are also a function of age. Youths make more friends but are more willing to lose them while adults make less friends and are always under the pressure of keeping them. The use of social drugs is another factor of lifestyle that depends on age. While youths are under pressure to select from numerous options, adults are already condemned to their addiction. Finally, while youths are making adventures into economic conundrum, adults are already wallowing in perpetual poverty or plenty. By these, we are now aware that most life events are age dependent.

\section{Life Styles}

Lifestyles, in my words may be defined as those human habits developed in the process of performing the characteristics of life. Some lifestyles are collective as in a cultural way of doing things, on the other hand, some are individualistic as in those individual habits that may be relatively distinct from the general way.

More scholarly, lifestyle is made up of the interests, opinions, behaviors, and behavioral orientations of an individual group, or culture [1-3]. Individualistic lifestyle is a reflection of attitudes, way of life and values possessed by the individual. It is therefore a means of forging a sense of self and to create cultural symbols that resonate with personal identity. Now, let us consider lifestyle with respect to feeding as a characteristic of life. The way different cultures feed vary. Also, different individuals feed differently. More so, what different cultures and individuals eat differ.

\section{Effects of Lifestyles on Health in Relation to Age}

The effects of interaction between lifestyle and age are overwhelming. For example, the process of respiration is reversed when an individual inhales cigarette smoke and this phenomenon produces very harmful effects over time (as age of the user increases). In feeding, most people believe that it must be high calorie food or nothing. With our lifestyle here, we consume so much calories neglecting the effects of herbs, vegetables and fruits on the body. We do not need the calories at later age be our insulin level usually depreciates with age. Most herbs vegetables and fruits are medicines and they do so much beneficial works in the body. More so, a good knowledge of when to drink water cannot be over emphasized.
Excretion is the removal of waste products of metabolism from the body. The process gives so much work to the kidney, liver, lungs and skin. This is actually a function of age. To reduce the concentration and, hence, assimilation of toxic elements in the body, optimal amount of water is required in the body. Of enormous importance is an individual's sexual habit. A man is frequently woken from sleep by a strong phallus. By this, the body is saying, "I am ready, I need it, give it to me." But most men work themselves to tiredness and forget to perform their sexual responsibilities. Let me emphasize here that sexual intercourse is the only exercise that has impact on every cell of the body. Sex is a tranquilizer to the body, it helps in the release of essential hormones (endorphins) that reduce pain, hypertension, trigger positive feelings and enhances sleep [4]. It has been suggested historically that infrequent sex can aggravate the hyperplasia (prostatitis) [5,6]. In our effort to balance between individual attributes, behviour and achievement with societal expectations, we put so much mental and psychological burdens on ourselves. This, in relation with age, wears down one's self esteem and can ultimately lead to mental and psychological disorder.

When we are sick, we get too desperate to get well again to the extent that we take medication from wherever it comes from. While drugs/medications are produced with primary aim of treating illnesses, drugs are poisonous and their use is dangerous when not properly administered. To this end, I see medication as the process of using what would kill us in the future to treat what could have killed us now.

When we drink alcohol and boos, we should recall that alcohol has other effects on the body than giving us some sense of euphoria. When excessively taken, it is toxic and should be treat accordingly. Continual use can cause organ damage and loss of self-esteem.

Effective lifestyles for healthy life above forty years are those lifestyles that should be adopted in order to achieve a complete wellbeing to the individual above that age. These, from the foregoing, include but not limited to, proper choice of what to eat and what to avoid; proper choice of life partners; regular, enjoyable and satisfactory sexual life; planning of economic and financial life; avoiding illicit drugs and drug/substance abuse; adopting good stress management strategies and others.

\section{Acknowledgement}

None.

\section{Conflict of Interest}

No conflict of interest.

\section{References}

1. Webster.com (2018) webster.com/dictionary/lifestyle. Retrieved from Merrian-Webster's Dictonary: www.webster.com

2. Lynn RK, Angeline GC (2011) Consumer Behavior Knowledge for Effective Sports and Event Marketing. New York: Routledge, USA

3. Spaergaren G (2011) Lifestyle, Consumptionand the Environment: The Ecological Modernisation of Domestic Consumption. Environmental Politics 9(1): 50-75. 
4. Web Med (2018) Exercise and Depression. Web Med.

5. Jacobsen ST, Jacobsen DJ, Rohe DE, Girman CJ, Roberts RO, et al. (2003) Frequency of Sexual Activity and Prostatic Health: Facts or Fairy Tale? Urology 61(2): 348-353.
6. Kok ET, Schouten BW, Bohnen AM, Groeneveld FP, Thomas Sa (2009) Risk Factors for Lower Urinary Tract Symptoms Suggestive of Benign Prostatic Hyperplasia in a community-based Population of Aging Men: the Krimpen Study. J Urology 181(2): 710-716. 